\title{
Simulación y desarrollo de horno termo solar para el cocimiento de alimentos
}

\section{Simulation and development of solar thermal oven for food cooking}

\author{
LINARES-ENRÍQUEZ, Alejandro†*, GARDUÑO-DÍAZ, Aldair Renato, NIETO-TRUJILLO, Héctor \\ Aaron y GARCÍA-ÁVILA, Jorge Luis
}

Tecnológico de Estudios Superiores de Tianguistenco, México.

ID $1^{\mathrm{er}}$ Autor: Alejandro, Linares-Enríquez / ORC ID: 0000-0002-2250-0730, Researcher ID Thomson: F-8123-21018, CVU CONACYT ID: 607628

ID $1^{\mathrm{er}}$ Coautor: Aldair Renato, Garduño-Díaz / ORC ID: 0000-0002-7657-7792

ID $2^{\text {do }}$ Coautor: Héctor Aaron, Nieto-Trujillo / ORC ID: 0000-0001-5703-2327

ID $3^{\text {er }}$ Coautor: Jorge Luis, García-Ávila / ORC ID: 0000-0002-4403-0266

DOI: $10.35429 /$ JOTI.2020.13.4.12.20

Recibido 15 Enero, 2020; Aceptado 30 Junio, 2020

\section{Resumen}

Este proyecto implementa un horno termo solar para el cocimiento de alimentos con base a radiación solar para generar el calor y la transformación de los alimentos para su consumo y, con ello tener una propuesta sustentable de energía renovables con el aprovechamiento de la energía solar, una entrada a la sustentabilidad de acuerdo a los objetivos de sostenibilidad que se requiere para nuestro país; el prototipo de horno establece rentabilidad económica, ya que no se tendrá un gasto por algún tipo de combustible, permitiendo tener una vida más ecoamigable con el medio ambiente, lo cual nos permitirá una alternativa enfocada al cuidado del planeta, el proyecto es un horno termo solar para la cocción de alimentos, a través del uso de la energía solar, específicamente de la radiación y transferencia de calor, la cual obtiene la temperatura con un sistema termo solar impulsado por una bomba con una alta presión, la cual moverá un fluido térmico a través de un serpentín el cual rodea al horno por dentro y siguiendo la base de un captador hacer una recirculación del mismo el cual evitaría el aumento de costo en uso de gas LP o natural e incluso leña, que representa dinero invertido para ciertos sectores de la población.

\begin{abstract}
This project implements a solar thermal oven for cooking food based on solar radiation to generate heat and transform food for consumption and, with it, have a sustainable proposal for renewable energy with the use of solar energy, an entrance to sustainability according to the sustainability objectives required for our country; The oven prototype establishes economic profitability, since there will be no expense for any type of fuel, allowing a more eco-friendly life with the environment, which will allow us an alternative focused on caring for the planet, the project is a solar thermal oven for cooking food, through the use of solar energy, specifically radiation and heat transfer, which obtains the temperature with a solar thermal system powered by a pump with a high pressure, which will move a thermal fluid through a coil which surrounds the oven from the inside and following the base of a sensor make a recirculation of the same which would avoid the increase in the cost of LP or natural gas and even firewood, which represents money invested for certain sectors of the population.
\end{abstract}

Oven, Solar thermal, Cooking

Horno, Termo solar, Cocimiento

Citación: LINARES-ENRÍQUEZ, Alejandro, GARDUÑO-DÍAZ, Aldair Renato, NIETO-TRUJILLO, Héctor Aaron y GARCÍA-ÁVILA, Jorge Luis. Simulación y desarrollo de horno termo solar para el cocimiento de alimentos. Revista de Invención Técnica. 2020. 4-13:12-20.

\footnotetext{
*Correspondencia al Autor (Correo Electrónico: alejandro.linares@test.edu.mx)

$\dagger$ Investigador contribuyendo como primer autor.
} 


\section{Introducción}

En el desarrollo del proyecto se realiza el análisis de los componentes del horno dentro del diseño, la simulación y la conversión de energía, se definen los parámetros de entrada del sistema, donde la parte estructural y térmica es esencial en la obtención de transferencia de calor, estableciendo el comportamiento de funcionamiento del sistema termo solar que será el encargado del cocimiento de alimentos.

El desarrollo tecnológico ha permitido dar propuestas de las diversas formas para calentar o dar cocción a los alimentos, desde un principio una forma de cocinar alimentos es con fuego utilizando leña, pero esta forma ha causado la tala de millones de árboles de forma alarmante, impactando en el medio ambiente, por lo que es necesario buscar nuevas alternativas que puedan generar este mismo beneficio, cuidando el medio ambiente y los recursos con los que se cuenta, analizando su costo con los beneficios que aporta.

La energía solar es una fuente inagotable, por ello, en los últimos años ha tomado una relevancia dentro de la generación de energía eléctrica y calorífica, y al ser una fuente no convencional nos brinda una alta gama de posibilidades para dar uso instantáneo de formas convencionales y poder generar ingresos, el aprovechamiento de este tipo de energía puede darse para diferentes aplicaciones y para el cocimiento de alimentos es una alternativa.

Lo que se pretende con este proyecto, es construir un horno termo solar capaz de dar cocción a los alimentos, estableciendo capacidad térmica, cuál será la temperatura que nos permitirá alcanzar para determinar que alimentos se podrán cocinar, como verduras, carnes, pan, entre otros, estableciendo que se puede transportar fácilmente gracias a sus dimensiones y peso reducido, sus materiales son de bajo costo y presenta una nueva variante en su diseño.

Nuestra propuesta es determinar la funcionalidad del horno termo solar para el cocimiento de alimentos, analizando el diseño de fluido que realizara la transferencia de calor y determinando sus componentes mecánicos y eléctricos.
La energía solar es la fuente de energía de mayor disponibilidad en un amplio dominio de latitudes, con índices de incidencia máximos en zonas ecuatoriales y mínimos en regiones polares. Es una energía muy dispersa que puede alcanzar valores máximos de mil watts por cada metro cuadrado de superficie y varía en función de la época del año; es posible almacenarla y convertirla en calor y en electricidad, y ser aprovechada por sus efectos en materiales que presentan propiedades ópticas y eléctricas.

El objetivo principal de este trabajo es dar a conocer, que podemos utilizar un sistema termo solar para cocimiento de alimentos, que podemos aplicar esta energía a un fluido para transmitir calor y los beneficios que se tiene al aplicar este tipo de tecnología, considerando los principios del aprovechamiento de la energía solar térmica y cómo se puede aplicar en una gran diversidad de procesos, tanto domésticos, como comerciales e industriales.

La creación de un horno a base de radiación solar se realiza para poder tener una nueva manera más sana y mejor de obtener una mejor calidad de aire.

El colector termo solar se estructura de un sistema de tuberías con la cual transportara liquido calentado a través de un tanque colector, el fluido térmico esta almacenado y aislado para conservar el calor, después cuando se coloca algún alimento dentro del horno se inicia con el paso del fluido calentando la cámara del horno y cociendo los alimentos, que entraran por una primera y saldrán al final del horno, el horno contará con un aislante para que no se transmita energía al exterior, sino todo se concentre dentro de la cámara, también contara con una bomba para el impulso del fluido a través de un segundo colector, aprovechando al máximo la transferencia de calor, regresando al colector termo solar, contara de la misma manera con un sistema de monitoreo y control de temperatura de acuerdo a la temperatura que necesite el alimento en cocción.

\section{Desarrollo}

El aprovechamiento de las fuentes de energía renovables dejó de ser una ilusión para convertirse en una realidad para todos aquellos comprometidos con la protección del ambiente. 
Hay dos hechos que han cambiado radicalmente nuestra visión del pasado. En primer lugar, el hecho de que el petróleo se va a agotar, y somos testigos de los frecuentes aumentos en los costos de la gasolina y el gas. En segundo lugar, y tal vez más importante, el Cambio Climático Global, que está directamente relacionado con el alto consumo de hidrocarburos de cada nación del planeta, así como la colateral producción de gases de efecto invernadero en los que México contribuye con alrededor de $1 \%$. De aquí la propuesta del proyecto.

La creación de un horno funcional que trabajara con una energía renovable, siendo un eco-amigo ya que trabajara con celdas termo solares a través de la capacidad de colección y las cuales darán abastecimiento a un sistema de tuberías el cual rodeara el cajón del horno, seguida de una capa de aislante para poder retener el calor dentro de mismo, para que al final este tenga la capacidad de calentar y dar cocción a alimentos [5].

Para analizar cómo trabaja un sistema termo solar, debemos conocer las características de los fluidos térmicos para la transferencia de calor, comparando estas características con los alimentos que se colocaran en el horno para su cocimiento, por lo que, es uno de los sistemas energético donde podemos aplicar para reducir costos, con el consiguiente ahorro y disminución de emisiones de $\mathrm{CO}_{2}$.

Una instalación termo solar está formada por captadores solares, dos circuitos de fluido térmico, intercambiador de calor, acumulador y tuberías. La circulación del sistema se da por dentro de los circuitos, aprovechando la diferencia de temperaturas. Los colectores termo solares capturan la radiación solar para transformarla en energía calorífica.

El sistema solar térmico se compone principalmente de una cubierta de material transparente, generalmente se trata de vidrio, fibra de vidrio o fibra de cerámica, pero existen determinados plásticos que también se pueden utilizar con la ventaja de ser más baratos y manejables. La función de la cubierta es reducir al mínimo las pérdidas de calor por convección y radiación y por eso debe tener una transmitancia solar lo más alta posible.
Canal de aire, que es un espacio que separa la cubierta de la placa absorbente y puede estar vacío o no. Para calcular su espesor se deben equilibrar las pérdidas por convección y las altas temperaturas que se pueden producir si es demasiado estrecho.

Placa absorbente, es el elemento que absorbe la energía solar y la transmite al líquido que circula por las tuberías. La principal característica de la placa absorbente es que debe tener una gran absorción solar y una emisión térmica reducida. Para cumplir este requisito se utilizan materiales combinados para obtener la mejor relación absorción / emisión. Tubos o conductos, están tocando la placa absorbente para maximizar el intercambio de energía calorífica por conducción térmica. Por los tubos circula el líquido que se calentará y transportará el calor hacia el tanque de acumulación. Capa aislante, tiene la finalidad de recubrir el sistema para evitar y evitar pérdidas térmicas. Para que el aislamiento sea el mejor posible, el material aislante deberá tener una baja conductividad térmica.

Los sistemas de energía solar térmica resultan útiles en aplicaciones como el calentamiento de piscinas, para el uso doméstico, para usos industriales que requieren una mayor temperatura de $65^{\circ} \mathrm{C}$. La principal aplicación de la energía solar térmica es la producción de Agua Caliente Sanitaria (ACS) para el sector doméstico y de servicios. El agua caliente sanitaria se usa a una temperatura de 45 ${ }^{\circ} \mathrm{C}$, temperatura a la que se puede llegar fácilmente con solares planos que pueden alcanzar como temperatura media $80{ }^{\circ} \mathrm{C}$. La energía aportada por los captadores debe ser tal que en los meses más favorables aporte el $100 \%$ [4].

El resto de las necesidades que no aportan los captadores se obtiene de un sistema auxiliar, que habitualmente suele ser gasóleo, gas o energía eléctrica. Con este porcentaje de cubrimiento los periodos de amortización son reducidos. La energía solar térmica puede ser un complemento al sistema de calefacción, sobre todo para sistemas que utilicen agua de aporte a menos de $60{ }^{\circ} \mathrm{C}$. 
Una vez que sabemos que podemos aprovechar de esa energía, necesitamos elementos que permitan captarla, transportarla, cederla y acumularla. Un colector termo solar nos permite la transferencia de calor y acumulación de energía, nos permiten captar la radiación solar y calentar el fluido caloportador que circula por ellos. Los hay de diferentes tipos y con diferentes rendimientos, como se muestra en la figura $1[3]$.

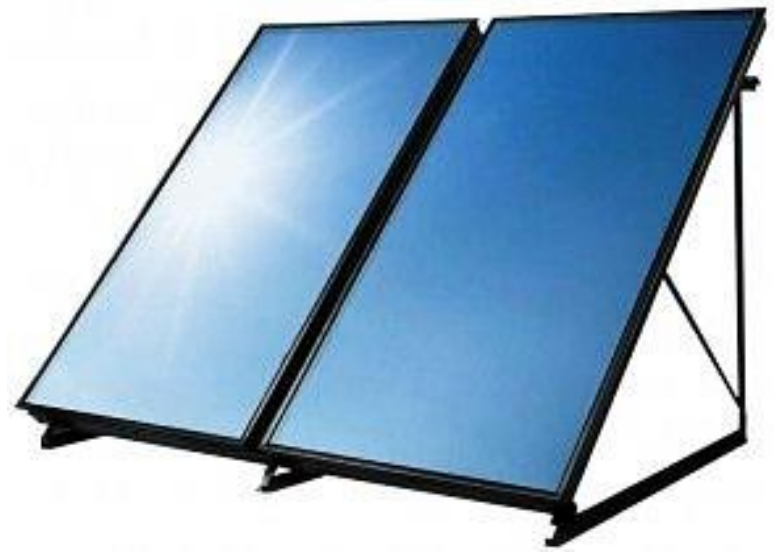

Figura 1 Paneles Solares Térmicos

Fuente: [3]

Establecer un circuito por donde transportar el fluido térmico en los captadores. Este circuito será cerrado. Por lo tanto, hablaremos de circuitos de ida y retorno. Podemos hacer un serpentín, en el que los captadores corresponderían a una cámara que caliente el fluido, y el circuito cerrado, a la ida y retorno de una instalación del colector como se muestra en la figura 2 .

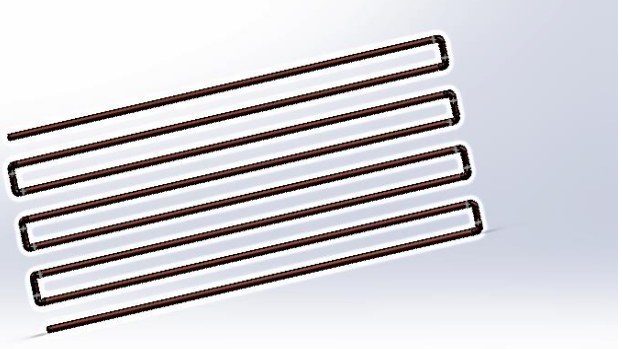

Figura 2 Tubería del sistema termo solar Fuente: Elaboración propia

En la instalación solar térmica, debemos ceder el calor transportado mediante un intercambiador de calor, esto se realiza en la cámara donde se meten los alimentos, cedemos el calor que será transmitido ya sea a una placa que se calentará o al espacio con el aire que se calentará, cediendo el calor a través de la trasferencia de este.
Los intercambiadores pueden ser externos al depósito, intercambiadores de placas, o interiores de serpentín.

La energía solar térmica, no se consume en su totalidad instantáneamente, ya que depende de la demanda existente en cada momento, por lo que, para no desaprovecharla, es necesario acumularla. Por ello, necesitamos un sistema de acumulación del fluido térmico, para que se pueda suministrar a medida que se va demandando. Esto se consigue con los Acumuladores o Inter acumuladores, que no son más que depósitos con capacidad y aislamiento suficiente para evitar, en lo posible, las pérdidas de energía.

En períodos de baja radiación térmica o con altas demandas de consumos, necesitaremos un sistema de apoyo que pueda cocinar los alimentos, independientemente del sistema solar, denominado sistema auxiliar. Por lo que podemos utilizarlo como un sistema hibrido utilizando algún combustible que caliente el horno y que pueda funcionar de manera convencional, esto dependiendo de las características de aplicación para el sistema termo solar en el cocimiento de alimentos [6].

\section{Metodología}

Para comenzar esta investigación se realiza con base al proyecto considerando la energía solar térmica, el cómo se fabrica un horno, cual material se ocupa, así como el desarrollo hablando de diseño, investigación y ensamble físicamente, para tener buenos resultados se modela y simula un horno termo solar tratando temas como son energías renovables, y sobre todo la energía solar la cual será la principal manera de obtener el calor para la cocción de alimentos, funcionando a través del aprovechamiento de las fuentes de energía renovables, el proyecto dejó de ser una ilusión para convertirse en una realidad para todos aquellos comprometidos con la protección del ambiente, como se muestra en la figura 3 [2]. 


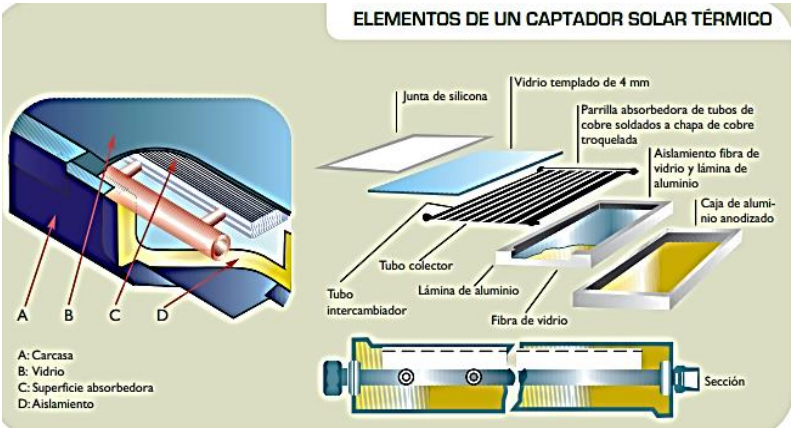

Figura 3 Elementos de un sistema solar térmico Fuente: [2]

En este caso más enfocado a la energía solar, el principio básico de funcionamiento de estos sistemas solares es sencillo, la radiación solar se capta y el calor se transfiere a un fluido, la cual será la energía primordial de obtener el calor para la cocción de alimentos funcionando, como se muestra en la figura 4 [2].

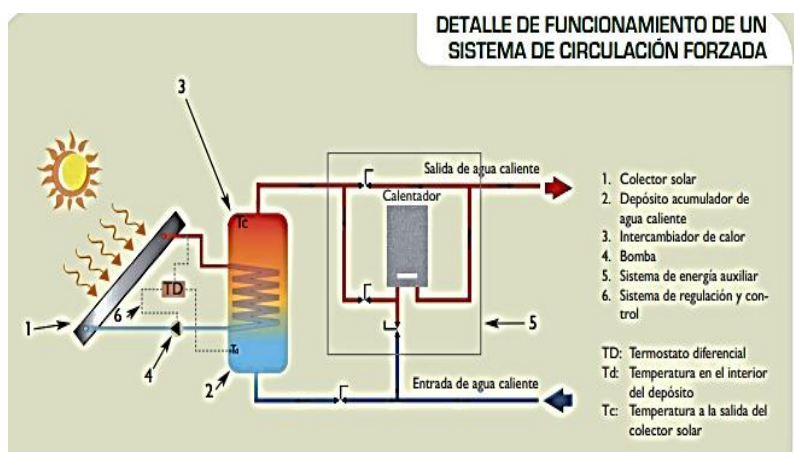

Figura 4 Sistema de circulación forzada Fuente: [2]

Para aprovechar la energía solar térmica se usa el captador solar, también denominado colector o placa solar. El fluido calentado se puede usar directa o indirectamente mediante un intercambiador de calor, en el caso del sistema termo solar el calentamiento del horno es a través de la transferencia de calor [2].

Para el análisis de la parte térmica debemos considerar las diferentes formas de transferencia de energía, como la conducción, que es la transferencia de energía de las partículas más energéticas de una sustancia hacia las adyacentes menos energéticas, como resultado de interacciones entre esas partículas.

La conducción puede tener lugar en los sólidos, líquidos o gases. En los gases y líquidos la conducción se debe a las colisiones y a la difusión de las moléculas durante su movimiento aleatorio.
En los sólidos se debe a la combinación de las vibraciones de las moléculas, ubicadas en posiciones más o menos fijas de una red cristalina y al transporte de energía por parte de los electrones libres y considerando la ley de Fourier de la conducción que es:

$\dot{Q}_{\text {cond }}=-k A \frac{d T}{d x}$

Aquí, $d T / d x$ es el gradiente de temperatura, esto es, la pendiente de una curva en un diagrama $T-x$, o sea la razón de cambio de $\mathrm{T}$ con respecto a la ubicación $x$. El calor es conducido en la dirección de la $T$ decreciente y el gradiente de temperatura se vuelve negativo al crecer $x$.El signo negativo garantiza que la transferencia de calor en la dirección de $x$ positiva sea una cantidad positiva.

La convección es un modo de transferencia de energía entre una superficie sólida y el líquido o gas adyacentes que están en movimiento y comprende los efectos combinados de la conducción y el movimiento de fluidos, por lo que se considera la Ley de Newton del enfriamiento.

$\left.\dot{Q}_{\text {conv }}=h A \dot{\left(T_{S}\right.}-T_{\text {fluıdo }}\right)$

La radiación es la energía emitida por la materia en la forma de ondas electromagnéticas o fotones, como resultado de los cambios en las configuraciones electrónicas de los átomos o moléculas. En los estudios de transferencia de calor es de interés la radiación térmica, que es la forma de radiación emitida por los cuerpos debido a su temperatura $[7,10]$.

Además, analizamos la radiación solar que se establece de tres formas distintas [1]:

Radiación directa: es la radiación que nos llega directamente del Sol; sin haber incidido con nada por el camino $\mathrm{y}$, por tanto, sin haberse desviado ni cambiado de dirección. Esta radiación es la que produce las sombras. Es el tipo de radiación predominante en un día soleado. 
- $\quad$ Radiación difusa: es la radiación que nos llega después de haber incidido con cualquier elemento de la atmósfera como polvo, nubes, contaminantes, entre otros, por lo que ha cambiado de dirección. Es el tipo de radiación predominante en un día nublado.

- Radiación reflejada o albedo: es la radiación reflejada por la superficie terrestre; cobra importancia en las zonas con nieve, con agua (como cerca del mar o de una presa) o cualquier otra zona donde la reflexión sea importante.

- La radiación global: es la suma de la radiación directa y la radiación difusa.

Para el análisis se realizará a través del programa Workbench del programa computacional Ansys donde se realizará tanto el análisis estructural, como el análisis dinámico del mismo usando de primera mano para hacer el dibujo SolidWorks para posteriormente trasladarlo al primer programa computacional donde se realiza el análisis del elemento finito y el análisis fluid, como se muestra en la figura 5, donde está el serpentín que se colocara dentro del horno, así como en la figura 6 , donde se muestra que el sistema termo solar está colocado dentro de la cámara del horno para que entre estos dos elementos contemos con el sistema aislante.

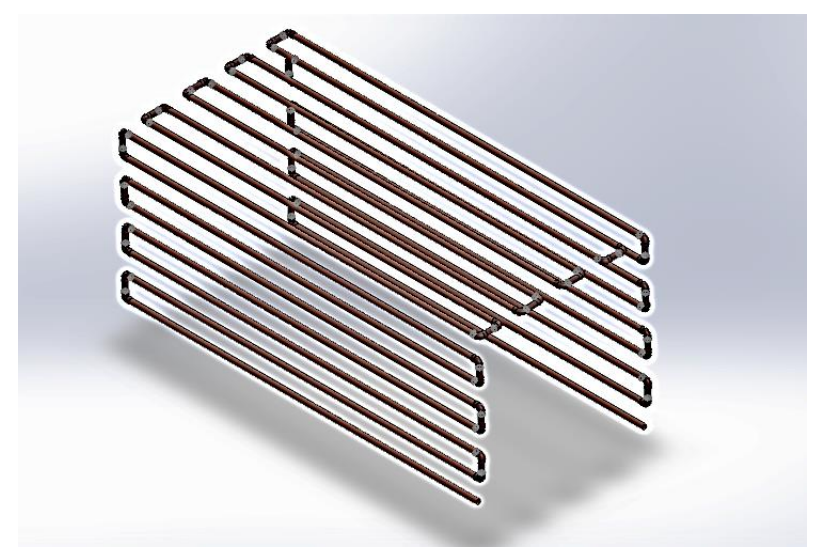

Figura 5 Sistema termo solar Fuente: Elaboración propia

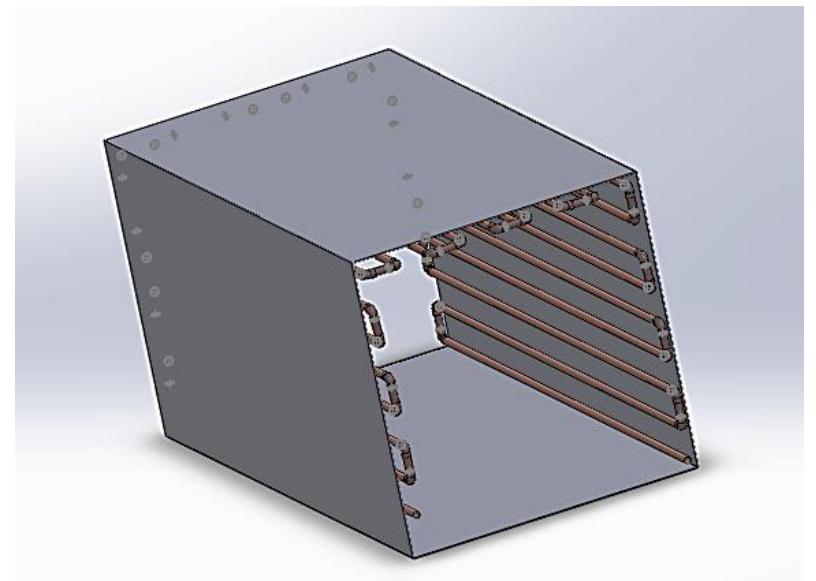

Figura 6 Horno termo solar Fuente: Elaboración propia

Se realizó la base del horno para su análisis estructural y forma que sostendrá todos los elementos del proyecto, como se muestra en la figura 7.

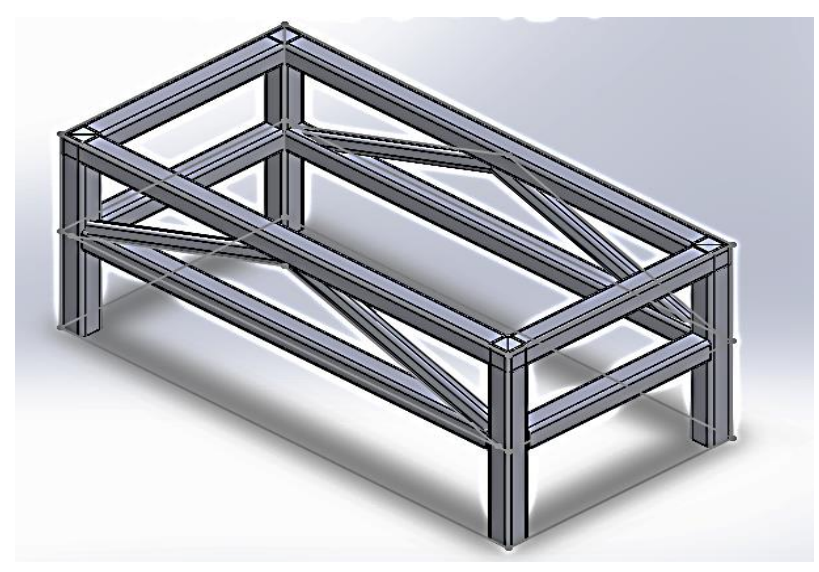

Figura 7 Base del Horno termo solar Fuente: Elaboración propia

Con el análisis estructural del sistema termo solar, se desarrolló una simulación para el comportamiento térmico en las tuberías, considerando el aceite térmico TERMICAL-75, que contendrá nuestro sistema térmico, para lo cual se desarrollaron las diferentes simulaciones, como se muestra el fluido extraído de una tubería y se muestra en la figura 8 . [9, 11]. 


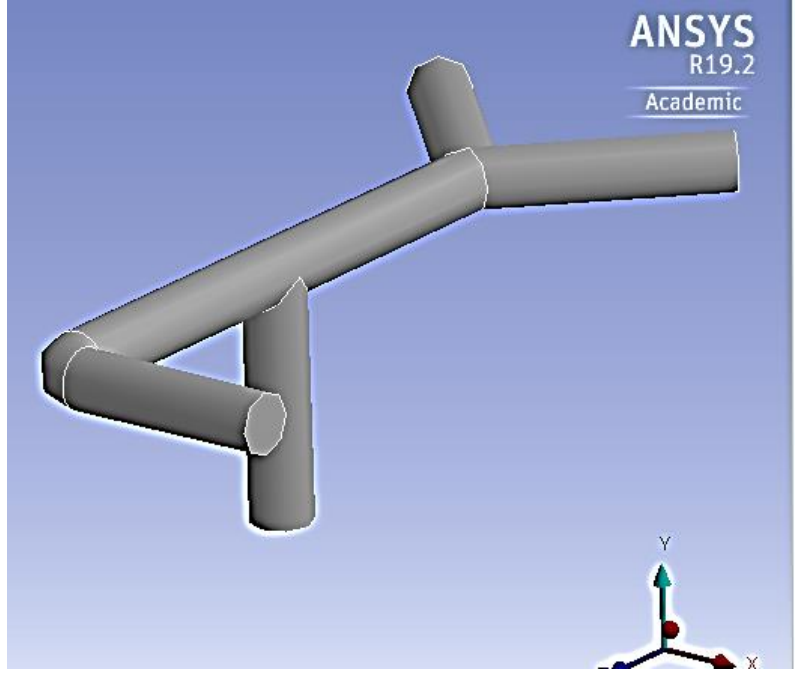

Figura 8 Sistema termo solar tuberías

Fuente: Elaboración propia

Con el sistema termo solar se desarrolla un análisis de elemento finito para determinar las características, como se muestra en la figura 9.

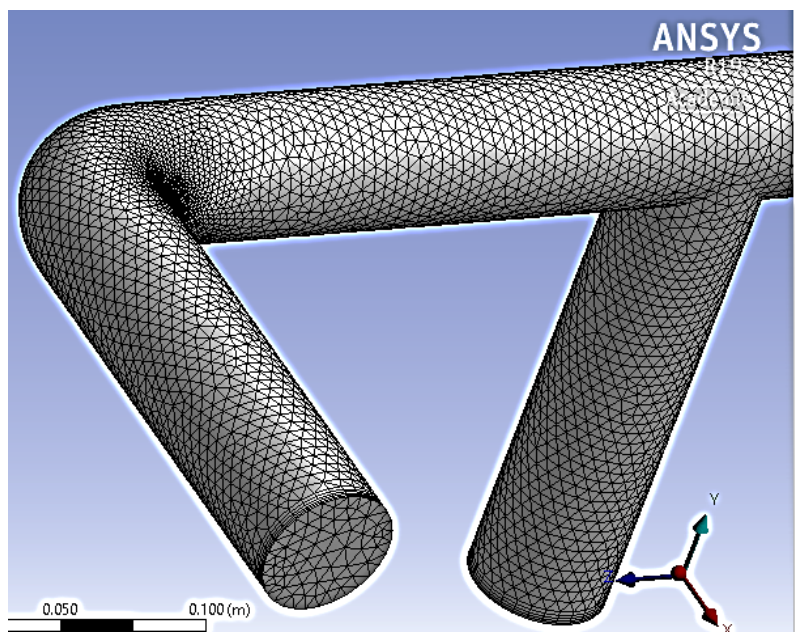

Figura 9 Análisis de elemento finito sistema termo solar, tuberías

Fuente: Elaboración propia

Se realiza a través de la simulación el comportamiento de tubería de acuerdo con las temperaturas de los fluidos por lo que se realizan análisis de Fluid Ansys, como se muestra en las dos imágenes de las figuras 10, 11 [9].

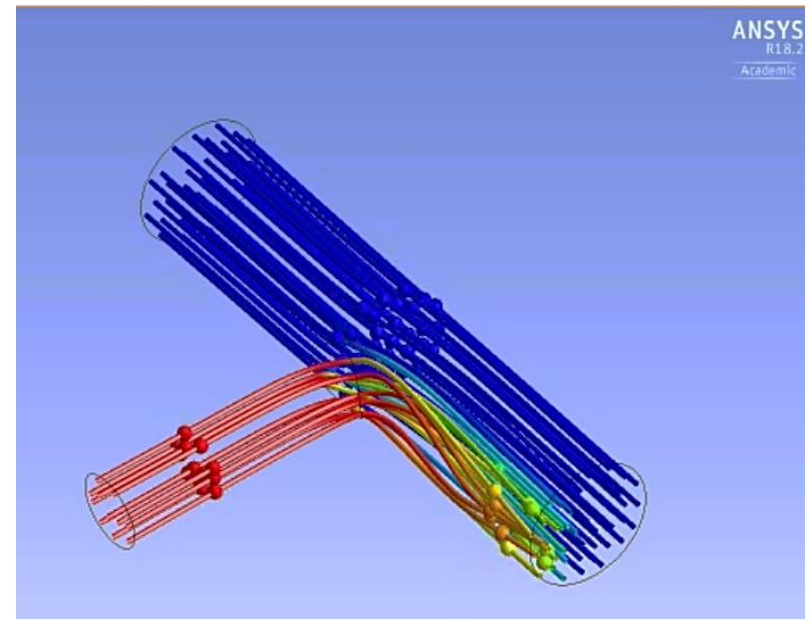

Figura 10 Análisis fluid ansys del horno termo solar Fuente: Elaboración propia

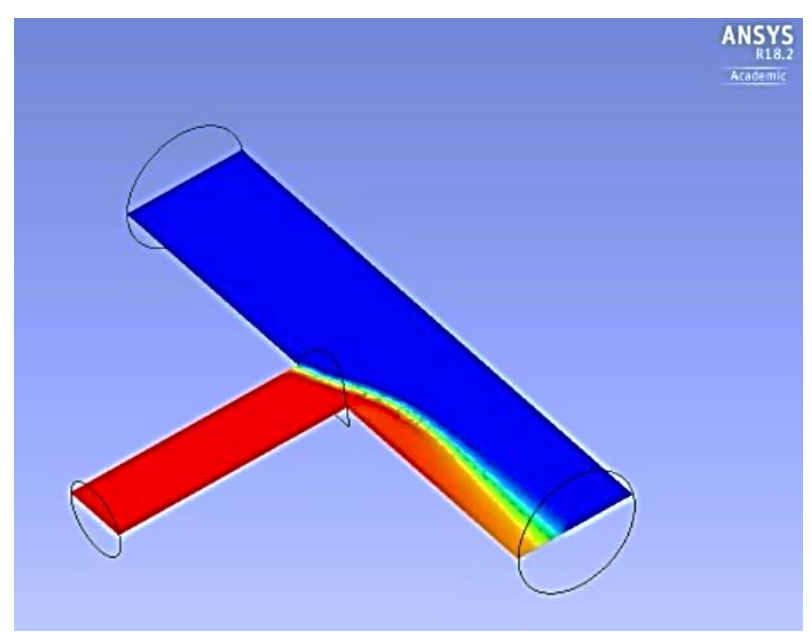

Figura 11 Análisis fluid ansys del horno termosolar Fuente: Elaboración propia

Se realiza una investigación y análisis para poder conocer las zonas correctas para poder comercializar, vender e instalar el horno, ya que el clima y zonas donde se coloca el horno es muy importante para la realización del proyecto. Se realizará la instalación de microcontroladores para poder regular la temperatura del horno y así poder tener una mejor funcionalidad de esta, y una investigación ardua para saber cuáles serán nuestros microcontroladores perfectos para el uso que se necesita. En cuanto al calor que se piensa obtener, se realizara una investigación más de cómo y cuáles serán las ecuaciones para usar ya que es muy importante saber esto para determinar el tubo correcto ya que será por donde circule el líquido que dará el calor necesario para la cocción de alimentos. 
En conclusión, se tendrá que el horno es una de las ideas más interesantes y difícil ya que tiene temas demasiado extensos lo cual nos dará mucha más información y más aprendizaje para poder dar un horno funcional y con mucha eficiencia.

\section{Resultados}

Dentro del desarrollo del prototipo de un horno termo solar para el cocimiento de alimentos, se comenzó con los avances de la simulación, para iniciar con la realización de la base del horno, como se muestra en la figura 12.
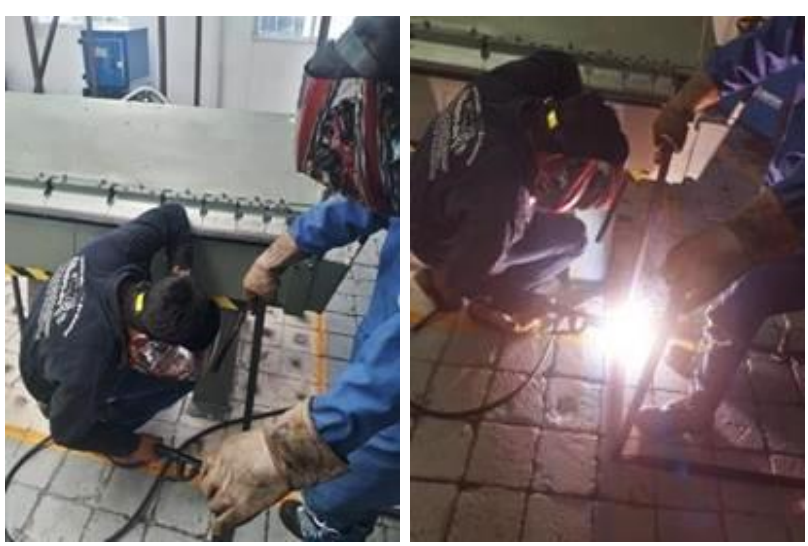

Figura 12 Inicio de la base del horno termo solar Elaboración propia

Con la base se realizó el horno termo solar donde se obtendrán diferentes pruebas y características de forma para la aplicación en alimentos, donde su base es de acero inoxidable ya que contendrá alimentos dentro, como se muestra en la figura 13 y figura 14.

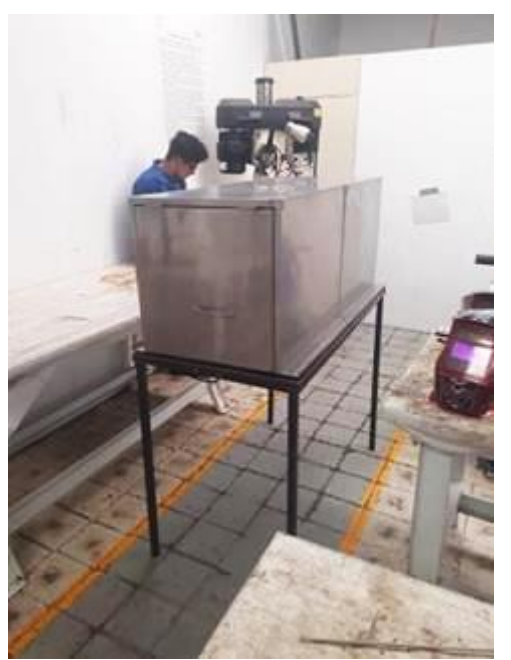

Figura 13 Horno termo solar Fuente: Elaboración propia

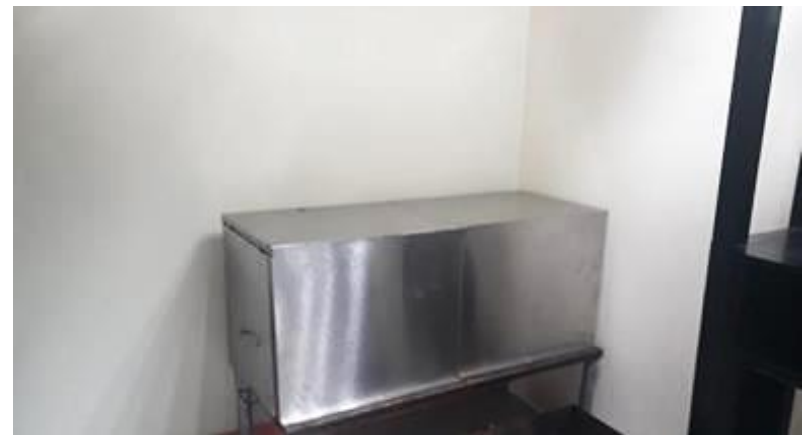

Figura 14 Horno termo solar Fuente: Elaboración propia

Sistema de tuberías. Con las dimensiones del horno termo solar, se inició con el sistema de tuberías, el cual estaría captando la energía solar que sería nuestro colector, así como el sistema que estaría dentro del horno en la parte de transferencia de calor, como se muestra en la figura 15.

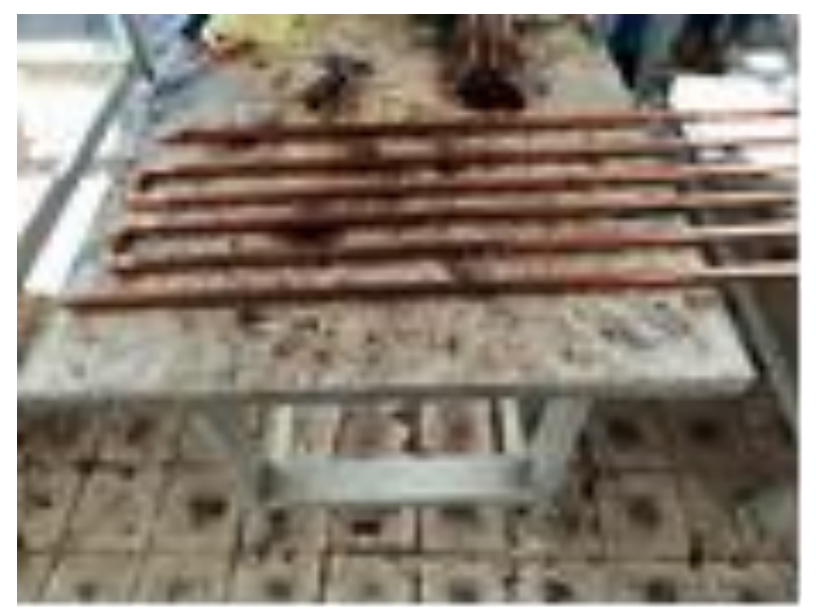

Figura 15 Sistema termo solar de tuberías Fuente: Elaboración propia

El sistema termo solar para el cocimiento de alimentos está en la etapa final con la colocación del sistema de monitoreo y control para su implementación e instrumentación del proyecto.

\section{Agradecimiento}

Al Tecnológico de Estudios Superiores de Tianguistenco el poder desarrollar el diseño, la simulación y propuesta de desarrollo del horno termo solar para el cocimiento de alimentos. 


\section{Conclusiones}

La simulación del horno termo solar para el cocimiento de alimentos permite establecer los elementos de análisis en su parte estructural y térmica y conocer las variables que serán importantes en la transformación de energía.

El análisis y diseño de los componentes que formaran el sistema termo solar son sistemas que deben ser analizados y evaluados, ya que podemos tener diferentes propuestas para mejorar estos elementos que ayuden al mejor aprovechamiento de la energía. Se determina con el proyecto que debemos establecer el intervalo de temperatura al cual trabaja nuestro proyecto para establecer cuales alimentos se podrán cocinar y que se tenga una buena cocción de alimentos dentro del prototipo armado.

Es necesario determinar diferentes fluidos que soporte más temperatura y que puedan ser utilizados en el sistema termo solar, para contar con mejor captación de calor dentro del horno. Determinar el funcionamiento y recorrido optimo del fluido dentro del sistema termo solar, esto referido a que el fluido se encuentre a una velocidad constante, de mantenga a una temperatura ideal para el alimento y obtener calor perdido a través de otra alternativa, obteniendo la transferencia de calor en un $100 \%$.

Es un proyecto de altos recursos económicos pero que obtendrá ciertos beneficios a largo tiempo como los es: ahorro en combustible, disminución de la contaminación eólica, etc. El proyecto establece, desarrolla y muestra la importancia del diseño, la simulación y el control dentro de la ingeniería para los sistemas de transferencia y aprovechamiento de energía, por lo que los resultados obtenidos demuestran el funcionamiento y la operación del sistema termo solar.

\section{Referencias}

[1] Rodríguez, J. and Hernández, C., (2008). Energías Renovables y Eficiencia Térmica. 1st ed. CANARIAS: Instituto Tecnológico de Canarias, S.A., pp.45-61.
[2] García Valladares, O. and Pilatowsky Figueroa, I., (2017). Aplicaciones Térmicas De La Energía Solar En Los Sectores Residencial, Servicios E Industrial. 1st ed. México: Universidad Nacional Autónoma de México, Instituto de Energías Renovables, pp.101-134.

[3] https://instalacionesyeficienciaenergetica.co $\mathrm{m} /$ como-funciona-la-energia-solar-termica/

[4] https://solar-energia.net/energia-solartermica/baja-temperatura

[5] Richard G. Budynas y J. Keith Nisbett. (2012), Diseño de Ingeniería Mecánica de Shigley, Ed. Mc Graw Hill, 9na Edición.

[6] Ferdinand P.Beer, E Russell Johnston, Jr. (2004) Mecánica de Materiales, Tercera edición, Mc Grall Hill.

[7] CENGEL \& BOLES, (2010). Termodinámica. México. Mc Graw Gill

[8] Ansys, (2017). Inc. Manual ANSYS 18.2, 2017.

[9] Ansys, (2010). Inc. ANSYS FLUENT USER'S GUIDE, Release 13.0, November 2010.

[10] Buigues N. A. (2012). Desarrollo de un Sistema Alternativo de Medición Distribuida: Experiencia Piloto en un Horno Solar Híbrido. Avances en Energías Renovables y Medio Ambiente Vol. 16, 2012.

[11] Sánchez Medina, Fernando. (2016). Diseño y análisis de un pequeño horno solar con Tonatiuh, https://idus.us.es/handle/11441/50937. 